\title{
Multipactor suppression in dielectric-assist accelerating structures via diamondlike carbon coatings
}

\author{
Shingo Mori॰* and Mitsuhiro Yoshida ${ }^{\dagger}$ \\ KEK Accelerator department, Tsukuba, Ibaraki 305-0801, Japan \\ Daisuke Satoh \\ Research Institute for Measurement and Analytical Instrumentation (RIMA), \\ National Metrology Institute of Japan (NMIJ), National Institute of Advanced Industrial Science and \\ Technology (AIST), 1-1-1 Umezono, Tsukuba 305-8568, Japan
}

(Received 26 September 2020; accepted 25 January 2021; published 12 February 2021)

\begin{abstract}
A dielectric-assist accelerating (DAA) cavity is a standing-wave accelerating cavity that attains a Q-value of over 100,000 at room temperature by using the reflection of the dielectric layer; the DAA cavity is expected to be used with a small rf power source and high-duty operation. Thus far, the maximum accelerating field of DAA cavities has been limited to a few $\mathrm{MV} / \mathrm{m}$ by the multipactor discharges. Multipactor discharges are widely observed in the accelerator field, and their suppression has been studied to improve accelerator performance. By applying a diamondlike carbon (DLC) coating to reduce the secondary electron emission coefficient without sacrificing the Q-value of the cavity, we have demonstrated that a C-band rf pulse with a pulse length of $5.4[\mu \mathrm{s}]$ can be injected into a DAA cavity with a field of more than $10[\mathrm{MV} / \mathrm{m}]$ while suppressing the multipactor discharges.
\end{abstract}

DOI: 10.1103/PhysRevAccelBeams.24.022001

\section{INTRODUCTION}

The rf accelerating cavity is widely used to generate high-energy beams in numerous scientific and technological applications. The Q-value is a commonly used figure of merit for the power efficiency of the rf cavity and is based on the conductivity of the materials. The superconducting cavity has a high Q-value of greater than order $\mathcal{O}\left(10^{10}\right)$ at low temperature $T<4.2[\mathrm{~K}]$, while the normal conducting cavity has a Q-value of $\mathcal{O}\left(10^{4}\right)$ working at room temperature, which is determined by the ohmic loss of the metal.

A dielectric-assist accelerating (DAA) structure establishes a high Q-value of $\mathcal{O}\left(10^{5}\right)$ for C-band at room temperature reducing the magnetic field on the copper surface by inserting a dielectric cylindrical structure with low loss inside the copper cavity to constitute onedimensional photonic structure in the radial direction [1]. Assuming the infinite number of dielectric cells or neglecting the wall loss at the copper endplate, even a single layer of dielectric hollow cylinder enhances the Q-value up to

\footnotetext{
*smori@post.kek.jp

mitsuhiro.yoshida@kek.jp

†ai-satou@aist.go.jp
}

Published by the American Physical Society under the terms of the Creative Commons Attribution 4.0 International license. Further distribution of this work must maintain attribution to the author(s) and the published article's title, journal citation, and DOI.
$Q \sim 1.6 \times 10^{5}$ by reducing the magnetic field at the cylindrical copper wall. In the previous work [2], we fabricated DAA cavities with five accelerating cells (DAA5) to demonstrate the Q-value of $Q \geq 10^{5}$ at room temperature constituted by single-cell pillbox cavity of copper, the dielectric cylindrical structure, the dielectric disks. The special end-cell structure in DAA5 modifies the field configuration on the copper plates and reduces the effect wall loss from the endplate, which enables DAA5 to surpass the Q-value of $10^{5}$ by as few as five cells. Figure 2(a) shows a schematic figure of DAA5 with a $Q \sim 1.2 \times 10^{5}$, which is one order of magnitude larger than the typical Q-value of a normal conducting cavity.

In the case of DAA cavity without reducing the secondary electron yield (SEY) of the ceramics, the multipactor effect limits the maximum accelerating gradient up to approximately 1 or $2[\mathrm{MV} / \mathrm{m}]$. Considering the low-SEY coating to suppress the multipactor effect in the DAA cavity, the ideal coating for a DAA cavity should have both low electrical conductivity and low SEY. A coating of a material with high electrical conductivity, such as the TiN coating, reduces the Q-value of the DAA cavity by increasing the ohmic loss on the surface of the dielectric cells.

In this paper, we propose the low-SEY coating of the diamond-like carbon (DLC) known as hydrogenated amorphous-carbon (a-C:H).

Multipactor discharges are a widespread phenomenon in the accelerator field and have appeared in the development of $\mathrm{rf}$ windows, accelerator tubes, and beam pipes. In the 
multipactoring process, the number of electrons increases exponentially owing to repeated resonant collisions of electrons on the surface of a material in an alternating electric field; it occurs when the SEY of the material is greater than one.

In the accelerator community, the multipactor effect is suppressed by the following methods: (1) The coating of low-SEY material on the surface of the device reduces the multipactor effects, e.g., titanium nitride (TiN) coating applied for the rf windows [3,4], the SEY of the TiN and DLC coating on the alumina ceramics not only at room temperature but also at high temperature is studied [5], and the amorphous carbon is applied for the beam pipes [6-8]. (2) The grooved surface effectively reduces the SEY of the surface [9]. (3) One-point multipactor effect is eliminated by the use of an elliptical instead of the cylindrical shape for superconducting cavity [10]. (4) DC bias of a coaxial line suppresses multipactoring by avoiding the resonant condition [11]. (5) The external solenoidal magnetic field reduces the multipactoring loss in the dielectric-lined waveguide (DLW) $[12,13]$.

The maximum accelerating gradient of a dielectric-based accelerating cavity is limited by multipactor discharges and the breakdowns [14]. Numerous high-power tests of $\mathrm{X}$-band dielectric-loaded accelerating structures (DLA) have been performed, and accelerating fields of up to $20[\mathrm{MV} / \mathrm{m}]$ have been achieved by applying an axial magnetic field to suppress multipactor effects [13]. The study of photonic bandgap cavities using sapphire rods shows a maximum electric field of $19.1[\mathrm{MV} / \mathrm{m}]$, which is limited by breakdowns in triple junctions [15]. Then both the multipactor suppression and antibreakdown treatments are necessary to achieve a higher accelerating gradient than that of the normal conducting cavities.

In Sec. II, we describe the detail of the DLC coating used in our experiment and show the effect of the low-SEY coatings, i.e., the DLC coating and the TiN coating, to the Q-value of the DAA5. In Sec. III, we explain the setup of the high-power test and introduce the two-cell DAA cavity (DAA2) to study the effect of DLC coating in the highpower test. In Sec. IV, we explain two evaluation methods of the accelerating gradient from the observables in the experiment. In Sec. V, we show the results of the highpower test comparing the DAA2 with and without DLC coating. In Sec. VI, we summarize the results and discuss the possibility of the DAA cavity based on the multipactor suppression by the DLC coating.

\section{THE DIAMONDLIKE CARBON COATING TO REDUCE THE MULTIPACTOR EFFECT}

The coating to apply on the ceramics surfaces of a DAA cavity is required to have both a low electrical conductivity and a low SEY. Coating with conductive materials such as TiN is not favored, since it reduces the Q-value of the DAA by increasing the ohmic loss on the surface of the dielectric
TABLE I. The effect of TiN coating and DLC coating on the Q-value of the five-cell DAA cavity (DAA5).

\begin{tabular}{lclr}
\hline \hline Cavity & $f_{0}[\mathrm{MHz}]$ & $\beta$ & \multicolumn{1}{c}{$Q_{0}$} \\
\hline DAA5-1-uncoated & 5708.29 & 1.4 & 112000 \\
DAA5-1-TiN & 5713.01 & 0.79 & 64000 \\
DAA5-2-uncoated & 5717.10 & 0.93 & 113000 \\
DAA5-2-DLC & 5717.07 & 1.0 & 116000 \\
\hline \hline
\end{tabular}

cells. Table I in Sec. V shows that, when a TiN coating with a thickness of 10 [nm] was applied across the entire surface of the ceramic cells of DAA5, its Q-value is reduced by approximately 40 [\%]. The TiN coating has another issue in that discharges during the conditioning process will remove some amount (a few nanometers in thickness) of the coating material [16].

A diamondlike carbon (DLC) coating is a carbon coating of the amorphous structure [17]. The properties of DLC coating differ by the ratio of diamondlike $\left(\mathrm{sp}^{3}\right)$ bounds and graphitelike $\left(\mathrm{sp}^{2}\right)$ bounds, and the hydrogen content. Figure 2(e) shows the MgO cell of DAA2 after the DLC coating. The DLC coating was processed by the method called negative pulse biased ion beam plating method, by a company specializing in DLC coating [18]. This DLC coating on the dielectric cells has a thickness of $0.5[\mu \mathrm{m}]$, hydrogen content of 5-50[\%], and $\mathrm{sp}^{3} /\left(\mathrm{sp}^{2}+\mathrm{sp}^{3}\right)$ ratio of $20-50[\%]$, which is called hydrogenated amorphouscarbon (a-C:H). A similar method is studied in Ref. [19].

We focus on the DLC coating since it has low SEY on the ceramics cells. We measured the SEY of the DLC coating on an $\mathrm{MgO}$ sample with a thickness of $0.5[\mu \mathrm{m}]$ using an SEM-based device at KEK [20]. Figure 1 shows the measured SEY using the same definition as Ref. [20]. In the incident energies range between $0.5[\mathrm{keV}]$ and $2.0[\mathrm{keV}]$, the measured SEY monotonically decreases from 1.4 to 0.7 . The SEY was $\delta<1$, when the incident energy exceeds $1.1[\mathrm{keV}]$. Moreover, a diamondlike carbon

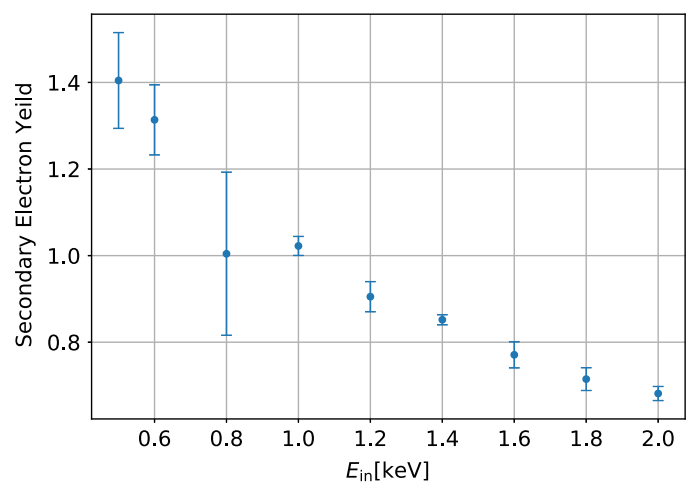

FIG. 1. The secondary electron yield (SEY) of the $\mathrm{MgO}$ samples with the DLC coating. The blue points denote the SEY of the DLC coating with a thickness of $0.5[\mu \mathrm{m}]$. The error bars denote the statistical error of $1 \sigma$. 
(DLC) coating is suggested to suppress the multipactoring than TiN coating for the normal-conducting cavity in $\mathrm{X}$-band [21].

\section{EXPERIMENTAL SETUP}

In this section, we introduce the experimental setup of the low-power measurement of the DAA5s and the DAA2s, and the high-power conditioning for the DAA2s.

We performed a low-power measurement for the DAA5s to investigate the effect of the ohmic loss of the coatings by comparing the Q-value before and after the coatings, since the Q-value of the DAA5s exceeding $10^{5}$ are sensitive to the conductivity of the coating on the dielectric cells. The low-power measurement is performed in the same setup as shown in the Ref. [2]. The Q-value is calculated from $S_{11}$ values obtained with the Agilent N5230A network analyzer.

In the previous work [2], we fabricated two combinations of DAA5 composed of six dielectric cells, which are labeled as DAA5-1, DAA5-2. Figure 2(a) shows the schematic figure of the sketch of the DAA5. We add the tags "-uncoated," "-TiN," and "-DLC" to indicate their surface conditions, where the thickness of the coating was $10[\mathrm{~nm}]$ for TiN and $0.5[\mu \mathrm{m}]$ for DLC, respectively. Since the DLC (a-C:H) coating has a high electrical resistivity at room temperature [22], its effect on the Q-value, when applied on the surface of dielectric cells, is expected to be negligible.
We fabricated a DAA test cavity composed of two cells (DAA2) to investigate the multipactor suppression for the DAA cavity by the surface treatment of the dielectric cells. We fabricated three combinations of dielectric cells labeled as DAA2-1, DAA2-2, and DAA2-3. To keep the translational symmetric field configuration shown in Fig. 2(b) and reduce the tangential electric field on the surface of the DAA cells, we did not adopt the end-cell structure which enhances the Q-value adopted in DAA5. The Q-value of DAA2 was $Q_{0} \sim 2.7 \times 10^{4}$, which is approximately 25 [\%] lower than the Q-values of the simulations assuming electrical conductivity $\rho=1.72 \times 10^{-8}[\Omega / \mathrm{m}]$ and dielectric loss $\tan \delta=6 \times 10^{-6}$. This is due to the insufficient contact among the copper cylinder, the copper coupler, and the copper endplates.

As shown in Fig. 2(c), the DAA2 is composed of the copper endplate, the copper cylinder, the coupler, the mode converter, and the dielectric cells. Figure 2(d) shows one of the examples of the $\mathrm{MgO}$ cell of DAA2 after machining with tolerances from $0.01[\mathrm{~mm}]$ to 0.07 [mm]. The $\mathrm{MgO}$ ceramics had the same characteristics as those used in the previous work [2], i.e., a relative permittivity of $\varepsilon_{r}=9.64$ and a loss tangent of $\tan \delta=6 \times 10^{-6}$.

Figure 2(f) shows the C-band rf system consisting of the power source, the waveguide, the acceleration cavity, and the measurement system on the test stand. The C-band rf was produced by a Tektronix TSG 4106A signal generator (SG) and amplified by a solid-state amplifier and a

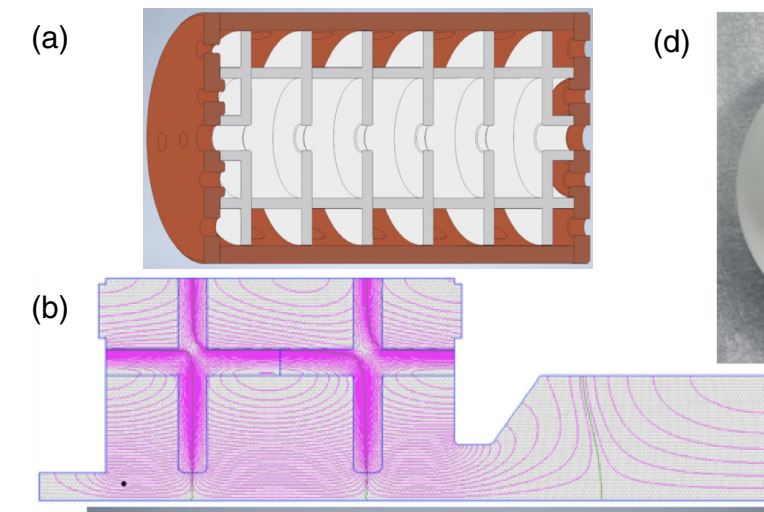

(c)

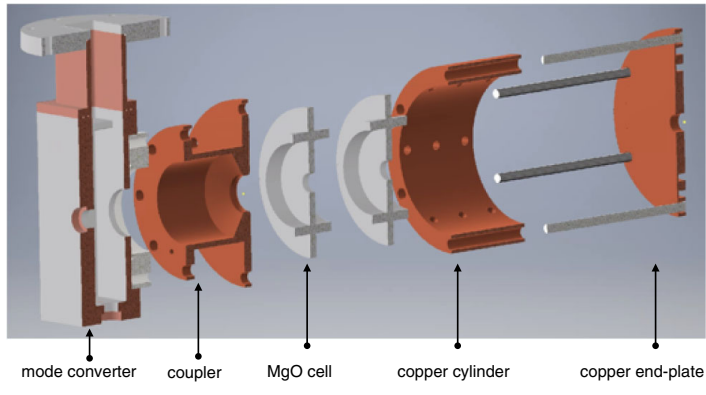

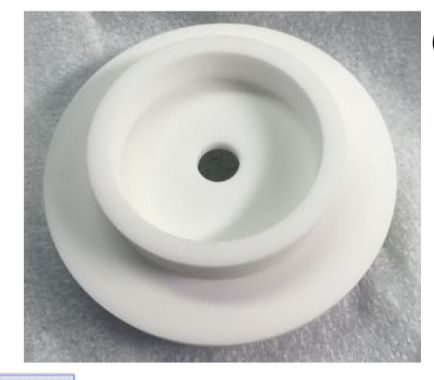

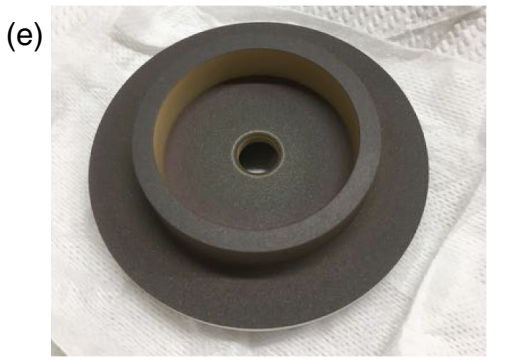

(f)
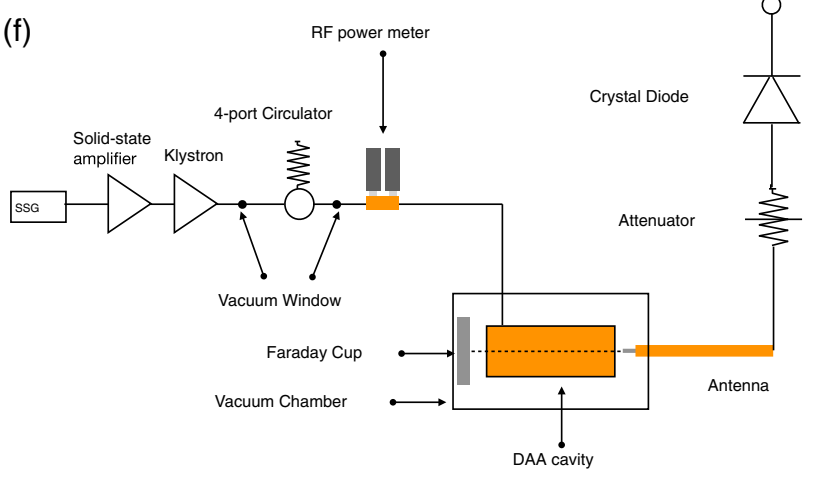

FIG. 2. (a) Schematic representation of the original DAA cavity with five cells (DAA5) [2]. (b) Cross section of the electric field of the accelerating mode calculated using SuperFish. (c) Development view of the two-cell cavity (DAA2). (d) MgO cell of DAA2 without coating and (e) with DLC coating with a thickness of $0.5[\mu \mathrm{m}]$. (f) rf system of the high-power conditioning from the klystron to the measurement of stored power through the antenna. 
klystron. The DAA2 was installed in the vacuum chamber and was connected to the rectangular waveguide via a mode converter inside the vacuum chamber. We placed a Faraday cup in front of the mode converter to measure the dark current that is emitted from the beam hole on the coupler side. We placed a pickup antenna which is composed of a semirigid coax cable outside the copper endplate, along the beam axis.

We performed the low-power measurement to obtain the Q-value and the coupling between the antenna and the accelerating mode by measuring the reflection coefficient, $S_{11}$, and the transmission coefficient, $S_{12}$. As shown in Fig. 2(f), the 4-port circulator is equipped with two vacuum windows. The section between them is kept at atmospheric pressure and a part of the 4-port circulator adjacent to the downstream vacuum window can be removed. Thus, we can perform the low-power measurements of the cavity in the vacuum chamber equipped with the antenna through the rectangular waveguide at the position of the downstream vacuum window.

The transmission coefficient $S_{12}$ from the waveguide to the antenna and a coaxial cable (Huber \& Suhner Sucoflex 104) can be measured to directly obtain information on the coupling between the antenna and the cavity, including the transmission loss of the coaxial cable. Neglecting the transmission loss by the rectangular waveguide, we can obtain the ratio of the power observed by the antenna via the coaxial cable and that stored inside the cavity from the transmission coefficient $S_{12}$.

In the high-power conditioning of the DAA2s, we measure the stored power in the DAA2 to estimate the accelerating gradient. The accelerating mode with the stored power weakly couples to the pick-up antenna, whose signal is finally read off from the oscilloscope. The small fraction of the accelerating mode is monitored by the antenna and transmitted through the cable, the attenuator (Lucas Weinschel Model 47), and a Keysight 8472B crystal diode, which generated a rectified voltage to be monitored by an oscilloscope (Iwatsu DS-5514). A measurement with the signal generator indicated that the attenuator damps the input rf power by $41[\mathrm{~dB}]$.

\section{EVALUATION OF ACCELERATING GRADIENT IN THE DAA CAVITY IN HIGH-POWER TEST}

In this section, we introduce the evaluation method of the average accelerating gradient $E_{z}(t)$ and its peak value $E_{z, \text { max }}$ from two methods using independent observables. Here, the time dependence means the transient behavior excited by the input rf pulse with period $T_{p}$.

The stored power $P_{c}(t)$ as a function of time can be evaluated via two independent methods using parameters obtained by the measurements from the low-power test. Below we will define $P_{c}^{A}(t)$ as the stored power evaluated by the readout from the antenna, and $P_{c}^{B}(t)$ as the stored power evaluated by the input rf pulse.

The first method evaluates the stored power $P_{c}^{A}(t)$ from the signal from the antenna considering the transmission coefficients $S_{21}$, the attenuator, and the crystal diode. Using the signal generator, we measured the relationship between the input power and the output voltage, $f_{\mathrm{dBm}}(\ln V)$, for the crystal diode and rf power meters, where $V$ is the voltage measured by the oscilloscope. Then, we can evaluate the stored power $P_{c}^{A}(t)$ as

$P_{c}^{A}(t)=1[\mathrm{~mW}] \times 10^{f_{\mathrm{dBm}}(\ln V) / 10} \times 10^{-S_{21}[\mathrm{~dB}] / 10} \times 10^{\Delta_{\mathrm{Att}} / 10}$,

where $\Delta_{\text {Att }} \sim 41$ is the power reduction measured by the attenuator.

The second method applies the transient model to compute the ideal stored energy $P_{c}^{B}(t)$ from the incident rf power $P_{\text {in }}(t)$ measured by the rf power meter, and the measured loaded Q-value $Q_{L}$. In the transient model, we assume $Q_{L}$ has a constant value, namely the time-varying multipactor effect is absent to cause power loss. By using the relation of power conservation $d W_{c} / d t=$ $P_{\text {in }}-P_{\text {ref }}-P_{c}^{B}, \quad Q_{0}=\omega W_{c} / P_{c}^{\mathrm{B}}, \quad P_{\text {in }}=G_{\mathrm{wg}} V_{\text {in }}^{2}, \quad P_{\text {ref }}=$ $G_{\mathrm{wg}}\left(V_{c}-V_{\text {in }}\right)^{2}, \quad P_{c}^{B}=\left(G_{\mathrm{wg}} / \beta\right) V_{\mathrm{c}}^{2}$, the cavity voltage $V_{c}$, and waveguide voltage follow

$$
T_{f} \frac{d V_{c}}{d t}+V_{c}=\frac{2 \beta}{1+\beta} V_{\mathrm{in}},
$$

where $T_{f}=Q_{L} /\left(\pi f_{0}\right)$ and $G_{\mathrm{wg}}$ denotes the waveguide admittance. We obtain the stored power $P_{c}^{B}(t)$ by substituting the measured time variation of the incident power $P_{\text {in }}(t)$ into Eq. (2).

Assuming the step function for the input rf pulse with the peak power $P_{\text {in }}$ and the pulse length of $T_{p}$, the stored power $P_{c}^{B}(t)$ satisfies

$$
P_{c}^{B}(t)=\frac{4 \beta}{(1+\beta)^{2}}\left(1-e^{-\frac{T_{p}}{T_{f}}}\right)^{2} P_{\mathrm{in}}
$$

Without multipactor effect, the gradient can be controlled by the magnitude of the input rf pulse and $T_{p}$ as long as $T_{p}<T_{f}$.

In general, the average accelerating gradient $E_{z}(t)$ can be evaluated from the stored power $P_{c}(t)$ and the shunt impedance per unit length $Z$ as $E_{z}(t)=\sqrt{Z \cdot P_{c} / L}$, where $L$ is the length of the cavity $L=52.485[\mathrm{~mm}]$. From the simulation using SuperFish, the fraction of shunt impedance per unit length in the Q-value is obtained as $\left(Z / Q_{0}\right)_{\mathrm{SF}}=4.25[\mathrm{k} \Omega / \mathrm{m}]$, which is determined only by the field configuration. To obtain the shunt impedance per unit length $Z$, we multiplied the fraction by the $Q_{0}$ obtained by the low-power measurement as $Z=\left(Z / Q_{0}\right)_{\mathrm{SF}} \cdot Q_{0}$. 
We define the average accelerating gradient $E_{z}^{A(B)}(t)$ as $E_{z}^{A(B)}(t)=\sqrt{Z \cdot P_{c}^{A(B)}(t) / L}$, and the peak value of the average accelerating gradient denotes $E_{z, \max }^{A(B)}=$ $\max \left(E_{z}^{A(B)}(t)\right)$.

\section{RESULTS AND DISCUSSION}

In this section, we show (1) the effect of the low-SEY coating on the Q-value from the result of the low-power measurement of the DAA5s, (2) the effect of the DLC coating on the multipactor suppression from the result of the high-power conditioning of DAA2.

\section{A. The Q-value of DAA5s}

First, we demonstrated that the DLC coating does not spoil the high power-efficiency of the DAA structure, by comparing the Q-value before and after the coatings, Table I shows the effect of TiN and DLC coatings on the Q-values of the two sets of DAA5, labeled as DAA5-1, DAA5-2. The results shown in Table I confirm that the DLC coating of $0.5[\mu \mathrm{m}]$ thickness on both sides of the ceramic cells caused approximately a 3[\%] change of the Q-value, which is considered within the error in lowpower measurements. On the other hand, the TiN coating with $10[\mathrm{~nm}]$ thickness reduces the Q-value by $40[\%]$.

\section{B. The high-power conditioning of DAA2s}

Second, we demonstrated that the DLC coating suppresses the multipactor effect in the DAA2s, namely the multipactor effect does not appear when the average accelerating gradient excesses $E_{z, \max }^{A}>1.6[\mathrm{MV} / \mathrm{m}]$. Before describing the high-power conditioning of DAA2s, we show the parameters obtained by the lowpower measurement which are measured in the vacuum chamber in the high-power test stand before starting the high-power conditioning.

The low-power measurements of the DAA2s provided parameters required to evaluate the average accelerating gradient $E_{z}^{A(B)}(t)$ as discussed in Sec. IV. In Table II, we show the obtained parameters. The three rows represent the coating conditions for three combinations of DAA2, i.e., without coating (DAA2-1-uncoated) and with DLC coating with a thickness of $0.5[\mu \mathrm{m}]$ (DAA2-2-DLC, DAA2-3DLC). The small variations of the measured resonant frequencies $f_{0}$ and the couplings through the iris of the

TABLE II. Results of the low-power test conducted using the network analyzer.

\begin{tabular}{lccccc}
\hline \hline Cavity & $f_{0}[\mathrm{MHz}]$ & $Q_{L}\left(\times 10^{4}\right)$ & $\beta$ & $Q_{0}\left(\times 10^{4}\right)$ & $S_{21}[\mathrm{~dB}]$ \\
\hline DAA2-1-uncoated & 5736.81 & 1.1 & 1.5 & 2.7 & 32.7 \\
DAA2-2-DLC & 5739.72 & 1.2 & 1.1 & 2.7 & 26.7 \\
DAA2-3-DLC & 5729.65 & 0.88 & 2.1 & 2.7 & 33.1 \\
\hline \hline
\end{tabular}

coupler $\beta$ comes from the small dimensional errors in the dielectric cells. The symbol $Q_{L}$ represents the loaded $Q_{L}=Q_{0} /\left(1+\beta+\beta_{\mathrm{a}}\right)$, where the coupling between the cavity and the antenna $\beta_{a}$ is negligibly small as compared to 1 .

In the high-power conditioning of DAA2s, the gradient of DAA2-2-DLC and DAA2-3-DLC increased by spending the number of shots and exceeds $10[\mathrm{MV} / \mathrm{m}]$, while one of DAA2-1-uncoated saturated around $1.6[\mathrm{MV} / \mathrm{m}]$ and was not improved by further conditioning. In Table III, we report the peak gradient $E_{z \text {,max }}^{A}$ as a typical value with a stable stored power $P_{c}^{A}(t)$ without the fluctuation by discharges after the conditioning. As for the DAA2 with DLC coating, the discharge with the large fluctuation of the stored power and the reflected power appeared at the higher gradient over the gradient shown in Table III. As further conditionings did not stabilize the waveforms fluctuated by the large discharges above the gradient shown in Table III, we consider the discharge effectively limits the maximum gradient of the current condition of DAA2s. After removing the DAA2s from the vacuum chamber, we found several traces of discharges on the edge of the dielectric cylinder as shown in Fig. 10.

We investigated the upper limit of the stored power by changing the incident power in the region between approximately $100[\mathrm{~W}]$ and $100[\mathrm{~kW}$. The repetition rate of the rf pulses is $50[\mathrm{~Hz}]$. During the high-power conditioning, we manually change the three parameters of the input rf pulse, namely the rf frequency, the magnitude of the incident power, and the pulse length, so as to maximize the peak of the stored energy in the cavity monitored by the pickup antenna. For each time after tuning the three parameters following the above criterion, we recorded ten samples of waveforms monitored by the oscilloscope by the repetition rate of $5[\mathrm{~Hz}]$.

The conditioning repeats two processes, namely modifying the three parameters and waiting until the fluctuation of the waveforms become stable. After modifying the parameters so as to enhance the peak gradient from $E_{z, 0}$ to $E_{z, 1}\left(E_{z, 0}<E_{z, 1}\right)$, the small fluctuation occurs at the pulse top of the waveform of the stored power $P_{c}^{A}(t)$ at which the gradient $E_{z}(t)$ exceeds the peak gradient $E_{z, 0}$ stabilized in the last step. Without the multipactoring effect, the fluctuation vanishes after tens of minites, and the peak gradient reaches $E_{z, 1}$. We call the fluctuation to be eliminated by the conditioning as the small discharges.

\section{The time-series data during the high-power conditioning}

The high-power conditioning spends several days with the repetition rate of $50[\mathrm{~Hz}]$ to reach the higher gradients. During the conditioning, we record the waveform of the oscilloscope after changing the parameter of the pulse generator or the power supply of the klystron. In each data taking, we record ten samples of waveforms with $5[\mathrm{~Hz}]$. 
TABLE III. The peak stored power $P_{c}^{A(B)}$ and average accelerating gradient $E_{z, \max }^{A(B)}$ of the sample shots shown in Fig. 9. The number of shot $N_{\text {shot }}$ denotes the number of rf pulse injected into the DAA2 during the conditioning until the sample shots.

\begin{tabular}{lccccc}
\hline \hline Cavity & $P_{c, \max }^{A}[\mathrm{~kW}]$ & $E_{z, \max }^{A}[\mathrm{MV} / \mathrm{m}]$ & $P_{c, \max }^{B}[\mathrm{~kW}]$ & $E_{z, \max }^{B}[\mathrm{MV} / \mathrm{m}]$ & $N_{\text {shot }}\left(\times 10^{6}\right)$ \\
\hline DAA2-1-uncoated & 0.89 & 1.6 & 85 & 16 & 1.5 \\
DAA2-2-DLC & 56 & 12 & 60 & 13 & 0.74 \\
DAA2-3-DLC & 48 & 12 & 40 & 11 & 3.8 \\
\hline \hline
\end{tabular}

In Figs. 3, 5, 4, we show the time series data of $E_{z, \max }^{A}, T_{p}$, $P_{\text {in }}^{\text {ave }}$ calculated from the recorded waveforms taken by the oscilloscope, respectively. The error bars in the plots denote the $1 \sigma$ region of the ten samples.

In the case of DAA2-2-DLC, the conditioning time required to reach an accelerating field of $10[\mathrm{MV} / \mathrm{m}]$ was approximately three hours, which is shorter than the conditioning time required to reach the same gradient for the normal-conducting cavity. In the case of DAA23-DLC, we increased the incident power over a longer period of time than in the case of DAA2-2-DLC. This is because we suppose the upper limit of the gradient $E_{z \text {,max }}^{A}$ would rise by spending more conditioning time at each incident rf power. However, as shown in Fig. 3 the upper limit of the gradient $E_{z, \max }^{A}$ is almost the same in both cases.

In Fig. 3, each point on the plot shows the mean value of the average accelerating gradient $E_{z, \text { max }}^{A}$ calculated by the peak value of the stored power $P_{c, \max }^{A}$. As we recorded those data points after changing the parameter of the input $\mathrm{rf}$ power, the data has large variations due to the small discharges inside the cavity.

Figure 4 shows the average value of the injected rf pulse $P_{\text {in }}^{\text {ave }}$ during the conditioning. We define $P_{\text {in }}^{\text {ave }}$ as the average of the $P_{\text {in }}(t)$ in the pulse top. In the conditioning of DAA22-DLC, we choose the parameters of the input rf pulse in

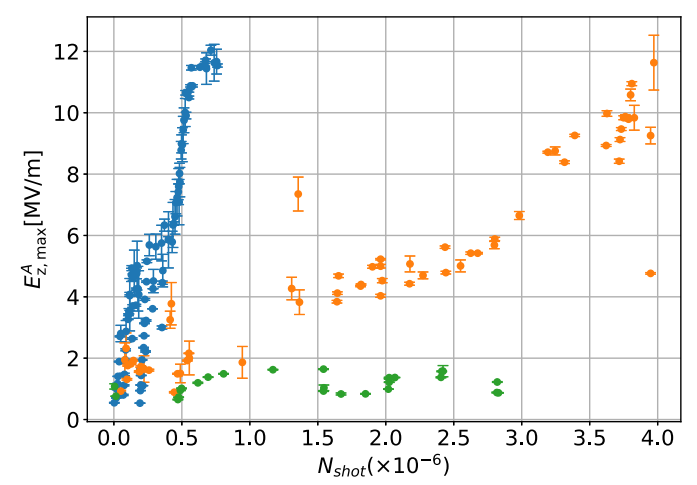

FIG. 3. The history of the peak value of the average accelerating gradient, $E_{z, \max }^{A}$, during the high power test for three cavities, DAA2-1-uncoated (green), DAA2-2-DLC (blue), and DAA2-3DLC (orange). The horizontal axes denote the number of shots of rf pulse injected to each cavity with a repetition rate of $50[\mathrm{~Hz}]$. The vertical axes denote the average accelerating gradient $E_{z, \max }^{A}$ at the peak of the stored energy measured by pickup antenna $P_{c, \max }^{A}$. the following manner usually applied for the conditioning of the normal conducting cavity. At first, keeping the pulse length shorter than the filling time $T_{f} \sim 0.5[\mu \mathrm{s}]$, we gradually increased $P_{\text {in }}^{\text {ave }}$ from $100[\mathrm{~W}]$ to $60[\mathrm{~kW}]$ which is required to excess $E_{z, \max }^{A}=10[\mathrm{MV} / \mathrm{m}]$ with larger pulse length. After the conditioning with the shorter pulse length, we went back to the low rf power and expanded the pulse length up to $T_{p} \sim T_{f}$ and gradually increased $P_{\text {in }}^{\text {ave }}$ with the same manner in the first step.

As long as the pulse length $T_{p}$ is smaller than the filling time $T_{f} \sim 0.5[\mu \mathrm{s}]$ in DAA2, the longer pulse length enhance the gradient as shown in Eq. (3). When $T_{p}>T_{f}$, the gradient has plateau region. Figure 5 shows the history of the pulse length $T_{p}[\mu \mathrm{s}]$ of the injected rf pulses. DAA2-3-DLC was stable with the pulse length of $5.4[\mu \mathrm{s}]$ and the accelerating gradient of $E_{z, \max }^{A}=11[\mathrm{MV} / \mathrm{m}]$. In the conditioning of DAA2-3-DLC, at first we kept the pulse length around $1[\mu \mathrm{s}]$ until the average accelerating gradient $E_{z, \max }^{A}$ reaches $10[\mathrm{MV} / \mathrm{m}]$, then we investigated the maximum pulse length keeping $E_{z, \max }^{A}>10[\mathrm{MV} / \mathrm{m}]$.

The history plot in Fig. 6 shows the peak value of the dark current $I_{d \text {,max }}$ recorded by the oscilloscope together with the rf pulses. The dark current is captured by the Faraday cup placed in front of the hole of the mode converter shown in Fig. 2(c). The Faraday cup is connected to the oscilloscope via the electric resistance of $100[\mathrm{k} \Omega]$. The peak value becomes large when the accelerating gradient becomes larger.

The eigenfrequency of the cavity is shifted from that obtained by the low-power measurement with frequency difference $\delta f_{\text {rf }}=f_{\text {rf,in }}-f_{\mathrm{LP}}$ because of the thermal expansion of the material and the plasma generated by the multipactor effect. Here $f_{\text {rf, in }}$ denotes the frequency of the input rf power, and $f_{\mathrm{LP}}$ denotes the eigenfrequency obtained by the low-power measurement shown in Table II. The history plot of the frequency difference during the high-power conditioning is shown in Fig. 7 and DAA2-1uncoated shows the upper shift of the frequency, while DAA2-2-DLC and DAA2-3-DLC show the lower shift of the frequencies. The multipactor effect in the metal cavity is known to enhance the eigenfrequency of the cavity [23], because the relative permittivity of the plasma is less than 1 .

For the case of DAA2-2-DLC and DAA2-3-DLC the multipactor effect was suppressed, since the frequency 


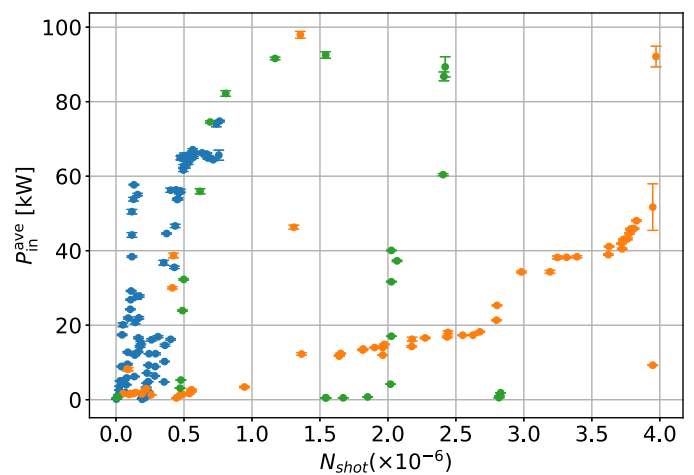

FIG. 4. The history of the injected power, $P_{\text {in }}$, during the high power test for three cavities, DAA2-1-uncoated (green), DAA22-DLC (blue), and DAA2-3-DLC (orange). The horizontal axes denote the number of shots of rf pulse injected to each cavity with a repetition rate of $50[\mathrm{~Hz}]$. The vertical axes denote the injected power, $P_{\text {in }}$, of the injected rf pulse.

shifts to the opposite direction from that of DAA2-1uncoated. We suspect that the frequency decrease comes from the thermal expansion of the dielectric cells. The thermal expansion of the dielectric cells would enhance the effective wavelength inside the cavity and the eigenfrequency of the cavity becomes smaller. Moreover in the DAA2s the heat generated by the power loss inside the dielectric cells could not propagate to the copper cylinder due to the poor thermal contact between them.

\section{The power fraction as a measure of discharges}

In Fig. 8 the discharge such as the multipactor happens at the points with smaller vertical value. The vertical value denotes the power fraction of the peak stored power $P_{c, \text { max }}^{A}$ in the average value of the injected $\mathrm{rf}$ pulse $P_{\mathrm{in}}^{\text {ave }}$, and without discharges the power fraction reaches 1 .

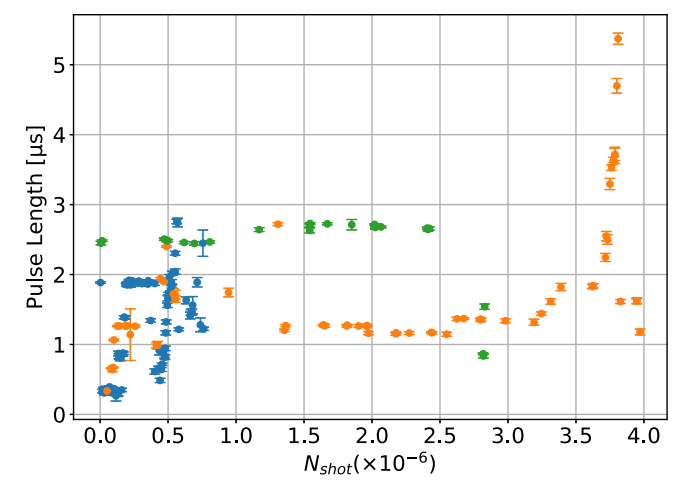

FIG. 5. The history of the pulse length, $T_{p}$, during the high power test for three cavities, DAA2-1-uncoated (green), DAA22-DLC (blue), and DAA2-3-DLC (orange). The horizontal axes denote the number of shots of rf pulse injected to each cavity with a repetition rate of $50[\mathrm{~Hz}]$. The vertical axes denote the pulse length, $T_{p}$, of the injected rf pulse.

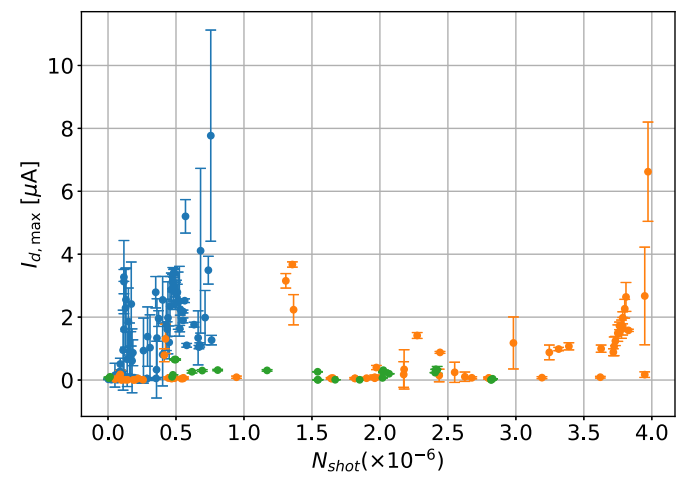

FIG. 6. The history of the peak value of the dark current, $I_{d \text {, max }}$, during the high power test for three cavities, DAA2-1-uncoated (green), DAA2-2-DLC (blue), and DAA2-3-DLC (orange). The horizontal axes denote the number of shots of rf pulse injected to each cavity with a repetition rate of $50[\mathrm{~Hz}]$.

The horizontal axis denotes the peak value of the accelerating gradient evaluated by $P_{c, \max }^{A}$, namely $E_{z, \max }^{A}$.

As a result of the multipactor in DAA2-1-uncoated, the power fraction becomes less than $10[\%]$ in the region between $0.65<E_{z, \max }^{A}<1.5[\mathrm{MV} / \mathrm{m}]$, since the multipactor blocks the input $\mathrm{rf}$ pulse filled inside the cavity.

For the case of DAA2-2-DLC, the conditioning process improved the power fraction $P_{c, \text { max }}^{A} / P_{\mathrm{in}}^{\text {ave }}$ from $15[\%]$ to 80 [\%]. At the beginning of the conditioning of DAA2-2DLC, the power fraction settled around 15 [\%] when increasing the input rf power from $1.3[\mathrm{~kW}]$ to $55[\mathrm{~kW}]$ keeping the pulse length of $0.35[\mu \mathrm{s}]$. In the second sweep we increased the input rf power from $0.1[\mathrm{~kW}]$ to $16[\mathrm{~kW}]$ with a longer pulse length of $1.8[\mu \mathrm{s}]$, then the peak gradient reached up to $5.8[\mathrm{MV} / \mathrm{m}]$ with high powerfraction of 80 [\%], then we stop the klystron for 4 hours. In the third step with $N_{\text {shot }}>0.4 \times 10^{6}$, we improved the gradient from $5.8[\mathrm{MV} / \mathrm{m}]$ to $12.3[\mathrm{MV} / \mathrm{m}]$ and the power fraction from $23[\%]$ to $75[\%]$.

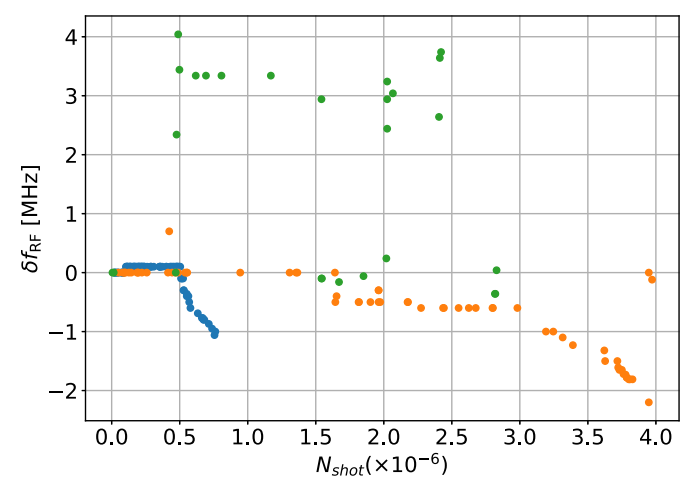

FIG. 7. The history of the rf-frequency difference from the initial rf-frequency, $\delta f_{\text {rf }}$, during the high power test for three cavities, DAA2-1-uncoated (green), DAA2-2-DLC (blue), and DAA2-3DLC (orange). The horizontal axes denote the number of shots of rf pulse injected to each cavity with a repetition rate of $50[\mathrm{~Hz}]$. 


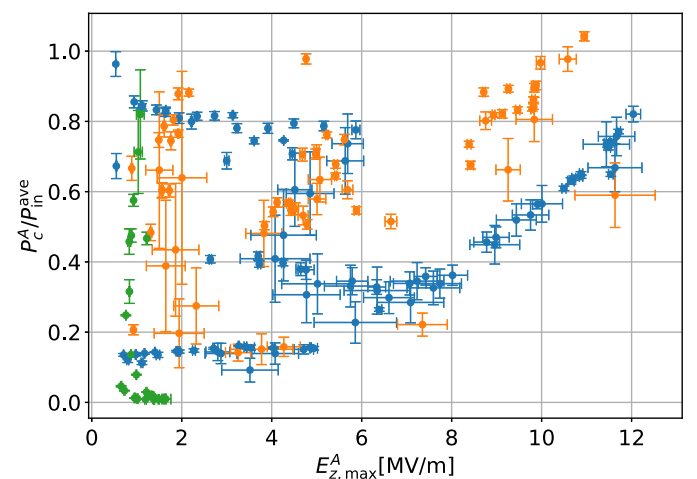

FIG. 8. The scatter plot of the peak accelerating gradient and the power fraction of the stored power to the input power, during the high power test for three cavities, DAA2-1-uncoated (green), DAA2-2-DLC (blue), and DAA2-3-DLC (orange).

In the conditioning of DAA2-3-DLC before $N_{\text {shot }}<$ $1.5 \times 10^{6}$, the discharges reduced the power fraction below 30 [\%] in the region $1.9<E_{z \text {, max }}^{A}<4.2[\mathrm{MV} / \mathrm{m}]$ and $E_{z, \max }^{A} \sim 7.4[\mathrm{MV} / \mathrm{m}]$. After that, the power fraction increased monotonically up to unity.

\section{E. The waveforms of DAA2s}

The absence of the multipactor effect in DAA2-2-DLC and DAA2-3-DLC appears in the comparison between $P_{c}^{A}(t)$ and $P_{c}^{B}(t)$, and in the waveform of the reflected power. Without the multipactor effect, we would obtain $P_{c}^{A}(t) \sim P_{c}^{B}(t)$ and the waveform of the reflected power exponentially dumps with a time constant $T_{f}$, which means the input rf power is absorbed in the cavity and eventually consumed by the cavity wall and the dielectric loss. When the multipactor effect appears, the additional power loss by the multipactor effect would cause the impedance mismatch. Then the most of the input power is reflected resulting in large reflected power without the absorption, and the stored power becomes small, i.e., $P_{c}^{A}(t) \ll P_{c}^{B}(t)$.

In Fig. 9, we show examples of the waveforms of the measured incident power $P_{\text {in }}(t)$ as blue lines, the measured reflected power $P_{\text {ref }}(t)$ as red lines, the stored power reconstructed by the pickup antenna $P_{c}^{A}(t)$ as yellow lines, and the stored power reconstructed by the incident power $P_{c}^{B}(t)$ as green lines. Figure 9 (left) denotes the result of DAA2-1-uncoated, Fig. 9 (center) denotes the result of DAA2-2-DLC, and Fig. 9 (right) denotes the result of DAA2-3-DLC. The corresponding peak values are shown in Table III, i.e., the stored power $P_{c, \max }^{A(B)}$ and the average accelerating gradient $E_{z \text {, max }}^{A}$ at the peak stored power.

In Table III, the multipactor effect can be seen from the comparison between $E_{z, \text { max }}^{A}$ and $E_{z, \text { max }}^{B}$. We compare the peak accelerating gradient estimated by two methods described in the previous section. The first row denoting DAA2-1-uncoated shows $E_{z, \max }^{A} \ll E_{z \text {, } \max }^{B}$, where the large difference comes from the multipactor effect on the dielectric surface without low-SEY coating. On the other hand, the second and third rows denoting DAA2 with the DLC coating show $E_{z \text {, } \max }^{A} \sim E_{z \text {, } \max }^{B}$, which means the multipactor effect is small or negligible.

The waveforms of DAA2-1-uncoated behaved differently below and above the gradient $E_{z, \max }^{A} \sim 1[\mathrm{MV} / \mathrm{m}]$. Below the gradient $E_{z \text {, max }}^{A} \sim 1[\mathrm{MV} / \mathrm{m}]$, the reflected power of DAA2-1-uncoated was consistent with the transient model in Eq. (2), while above that the multipactor effect modifies the reflected $\mathrm{rf}$ pulse reducing the absorption of the incident power in the cavity. When the multipactor effect saturates the stored power, the longer pulse length $T_{p}$ extends the plateau region of the stored power. As shown in the large difference between $P_{c}^{A}(t)$ and $P_{c}^{B}(t)$ in Fig. 9 (left), DAA2-1-uncoated did not absorb the input rf power after reaching $E_{z, \max }^{A}=1.6[\mathrm{MV} / \mathrm{m}]$.

Since the limit of the gradient at $E_{z, \max }^{A}=1.6[\mathrm{MV} / \mathrm{m}]$ in DAA2-1-uncoated is mitigated by the low-SEY coating in DAA2-2-DLC and DAA2-3-DLC, we consider the limitation stems from the multipactor effect on the surface of the dielectric cells in DAA structure.

As shown in the smaller difference between $P_{c}^{A}(t)$ and $P_{c}^{B}(t)$ in Fig. 9 (center,right), DAA2-2-DLC and DAA2-3DLC can suppress the multipactor effect by the DLC
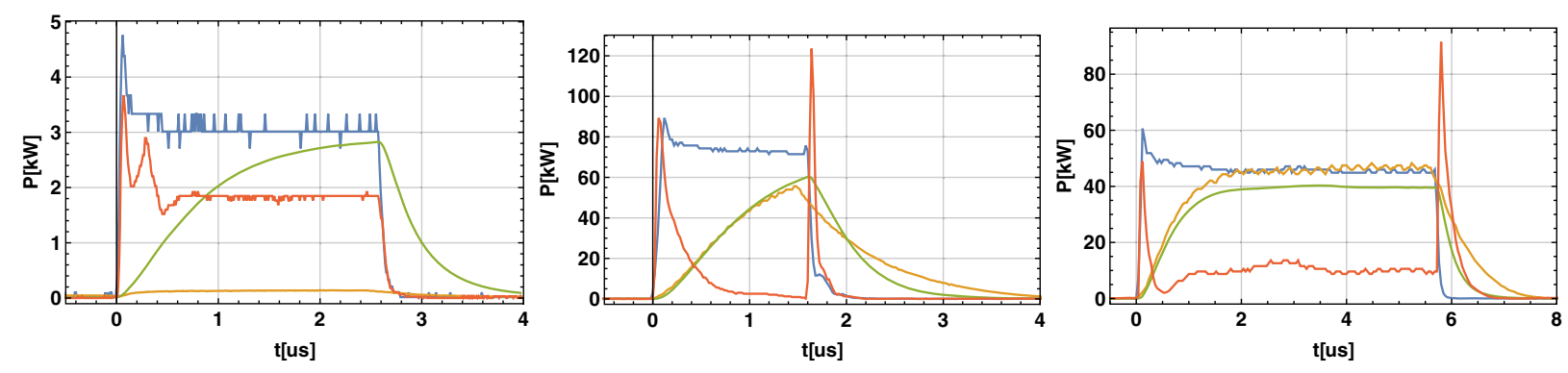

FIG. 9. Waveforms of the measured incident power (blue), reflected power (red), and the stored power reconstructed by the antenna (yellow), $P_{c}^{A}$, and by the incident power (green), $P_{c}^{B}$. For DAA2-1-uncoated (left), typical waveforms with multipactor effects are shown. For DAA2-2-DLC (center) and DAA2-3-DLC (right), the waveforms show stable stored power with an accelerating gradient exceeding $10[\mathrm{MV} / \mathrm{m}]$. 


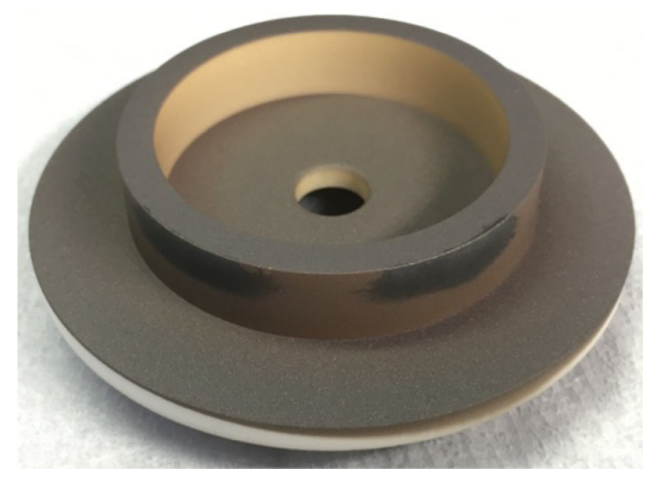

FIG. 10. The DLC-coated MgO cell of DAA2-1-DLC after the conditioning process. The traces of the breakdown were observed at the contact point between the dielectric cell and the adjacent dielectric cell and at the contact point between the dielectric and the copper end plate. Those traces are at the outside surface of the dielectric cylinder adjacent to the edge.

coating. The waveforms of the reflected power show the clear absorption of the input rf power in the DAA cavity as they reach near the bottom after the exponential dumping.

\section{F. The breakdown of DAA2 with the DLC coatings}

In our setup of DAA2s with the DLC coating, the peak accelerating field $E_{z, \max }^{A}$ is limited by the discharge breakdown at the gap at the edge of the dielectric cylinder. In the case of DAA2-2-DLC, the stored power gradually increased during the conditioning process until the gradient exceeds $E_{z, \max }^{A} \sim 12[\mathrm{MV} / \mathrm{m}]$. Over the gradient, the waveforms of both stored power $P_{c}^{A}(t)$ and the reflected power $P_{\text {ref }}(t)$ started to largely fluctuate by the discharge breakdown. In the case of DAA2-3-DLC, the stored power gradually increased until the gradient reaches $E_{z, \max }^{A}=10[\mathrm{MV} / \mathrm{m}]$. When the pulse length $T_{p}$ exceeds $5.4[\mu \mathrm{s}]$, the breakdown waveform appears as in the case of DAA2-2-DLC.

In the breakdown phase, the breakdown-like pattern appears in the latter part of the waveforms over some critical gradient $E_{z, c}$. In the former part of the waveforms below $E_{z, c}$, the waveforms are normal and behave exponentially as described in Eq. (3). Once the gradient exceeds the critical value $E_{z, c}$ with the corresponding critical pulse length $T_{c}$, the reflection waveform stops absorbing the input $\mathrm{rf}$ and sharply rises to the peak in less than 100 [ps] and then the peak value decreases. From the critical pulse length $T_{c}$, the stored power $P_{c}^{A}(t)$ behaves exponential dumping with a time constant of $T_{f}$ as if the input rf pulse vanishes at $T_{c}$. In our observation, the critical gradient $E_{z, c}$ was different for each shot, and moved around 10 [MV/m]. After the breakdown happens, further conditioning does not enhance the critical gradient and does not reduce the variance of the fluctuation of $E_{z, c}$.

As shown in Fig. 10, after the breakdown, spot-like traces appeared at the outer surface of the edge of the dielectric cylinder. With a small gap between cells, those spots are exposed to the high electric field with magnitude up to the same as the peak accelerating gradient depending on the width of the gap. Since the electric field lines inside the dielectric cylinder are compressed by a factor of $\varepsilon_{r}$, they overflow outside the cylinder when flowing from one cylinder to the adjacent cylinder across the vacuum gap. In the ideal limit of no gap as shown in Fig. 2(c), the magnitude of the electric field at the outer surface of the junction has 20 [\%] smaller electric field than the peak electric field on the axis, which means the average accelerating gradient of $E_{z, \max }=12[\mathrm{MV} / \mathrm{m}]$ corresponds to $|E| \sim 3.8[\mathrm{MV} / \mathrm{m}]$ around the spots. When there is a finite distance of gap, the fraction becomes large from 20 [\%] to around 100 [\%] depending on the distance due to the overflow of the field.

Therefore, we consider that the discharges at the cell junctions determine the maximum accelerating electric field in the DLC-coated DAA cavity. In order to obtain a higher accelerating field, countermeasures against breakdowns at the cell junctions are required. For example, methods of separating the dielectric cells from each other or separating the dielectric cells from the metal endplates have been proposed in Refs. [24,25].

\section{CONCLUSION}

The dielectric-assist accelerating (DAA) structure has a high Q-value of order $\mathcal{O}\left(10^{5}\right)$ at room temperature by reducing the ohmic loss on the copper surface by applying the dielectric cylinder of low-loss dielectrics. While the energy efficiency is high, the maximum accelerating gradient has been limited by the multipactor effect by a few $\mathrm{MV} / \mathrm{m}$. We found the low-SEY coating of the diamondlike carbon (DLC) known as hydrogenated amorphous-carbon $(\mathrm{a}-\mathrm{C}: \mathrm{H})$ can suppress the multipactor discharge in the DAA cavity without reducing the Q-value of the DAA cavity. We have demonstrated that the DAA test cavity with two cells with DLC coating for the entire surface of the dielectric cells can support the average accelerating gradient over $10[\mathrm{MV} / \mathrm{m}]$ for incident rf pulse length of $5.4[\mu \mathrm{s}]$. We have also demonstrated that due to the quite low resistance of the DLC, even a $0.5[\mu \mathrm{m}]$-thick DLC coating does not reduce the Q-value $\mathcal{O}\left(10^{5}\right)$ of a fivecell DAA cavity.

The accelerating gradient over $10[\mathrm{MV} / \mathrm{m}]$ is enough high to consider the application for the industry linac. The high energy efficiency of the DAA cavity is applicable as an electron accelerator for X-ray and RI production working with a smaller of power source than the normal conducting cavities. To utilize the high Q-value of the DAA cavity in those applications, the DAA cavity is required to work with smaller rf peak power and longer pulse length of several $\mu \mathrm{s}$. We demonstrated that the DLC coating enables us the operation mode. 
For the application of the DAA cavity to the accelerator facility, we need to increase the higher accelerating gradient, e.g., $30[\mathrm{MV} / \mathrm{m}]$. If such a high accelerating gradient is accomplished in the future, a linear accelerator facility such as free-electron laser facilities may be able to operate at room temperature without rf pulse compression by the SLED cavity.

\section{ACKNOWLEDGMENTS}

We would like to acknowledge Hiroyasu Ego for helpful discussions. We would like to thanks Yoichi Toge for English proofreading. This work was supported by JSPS KAKENHI Grant-in-Aid for Scientific Research (A) (No. 16H02134), JSPS KAKENHI Grant No. 19K20609, and Mitsubishi Heavy Industries Machinery Systems, Ltd. The data that support the findings of this study are available from the corresponding author upon reasonable request.

[1] D. Satoh, M. Yoshida, and N. Hayashizaki, Dielectric assist ac- celerating structure, Phys. Rev. Accel. Beams 19, 011302 (2016).

[2] D. Satoh, M. Yoshida, and N. Hayashizaki, Fabrication and cold test of dielectric assist accelerating structure, Phys. Rev. Accel. Beams 20, 091302 (2017).

[3] A. R. Nyaiesh, E. L. Garwin, F. K. King, and R. E. Kirby, Properties of thin antimultipactor TiN and $\mathrm{Cr} 2 \mathrm{O} 3$ coatings for klystron windows, J. Vacuum Sci. Technol. A 4, 2356 (1986).

[4] K. Kennedy, B. Harteneck, G. Millos, M. Benapfl, F. King, and R. Kirby, Tin coating of the pep-ii lowenergy aluminum arc vacuum chambers, in Proceedings of the 1997 Particle Accelerator Conference (Cat. No. 97CH36167), Vol. 3 (IEEE, New York, 1997), pp. 35683570 .

[5] S. Michizono, Y. Saito, Suharyanto, Y. Yamano, and S. Kobayashi, Secondary electron emission of sapphire and anti-multipactor coatings at high temperature, Appl. Surf. Sci. 235, 227 (2004).

[6] C. Y. Vallgren, G. Arduini, J. Bauche, S. Calatroni, P. Chiggiato, K. Cornelis, P. C. Pinto, B. Henrist, E. Métral, H. Neupert et al., Amorphous carbon coatings for the mitigation of electron cloud in the cern super proton synchrotron, Phys. Rev. Accel. Beams 14, 071001 (2011).

[7] C. Y. Vallgren, G. Arduini, J. Bauche, S. Calatroni, P. Chiggiato, K. Cornelis, P. C. Pinto, E. Métral, G. Rumolo, E. Shaposhnikova et al., Amorphous carbon coatings for mitigation of electron cloud in the cern SPS, in Proceedings of the International Particle Accelerator Conference, Kyoto, Japan (ICR, Kyoto, 2010).

[8] E. Shaposhnikova, G. Rumolo, K. Cornelis, J. Axensalva, J. Jiménez, M. Taborelli, P. Chiggiato, S. Calatroni, G. Arduini, C. Y. Vallgren et al., Experimental studies of carbon coatings as possible means of suppressing beam induced electron multipacting in the CERN SPS, Tech. Rep., 2009.

[9] M. J. Smith and G. Phillips, Power Klystrons Today (Research Studies Press, Taunton in Somerset, England, 1995).

[10] U. Klein and D. Proch, Multipacting in superconducting rf structures, in Proceedings of the Conference of Future Possibilities for Electron Accelerators, Charlottesville, WU B (1979) pp. 78-34.

[11] P. Ylä-Oijala and M. Ukkola, Suppressing electron multipacting in ceramic windows by dc bias, Nucl. Instrum. Methods Phys. Res., Sect. A 474, 197 (2001).

[12] A. Kulikov, A. Fisher, S. Heifets, J. Seeman, M. Sullivan, U. Wienands, and W. Kozanecki, The electron cloud instability at pep-ii, in PACS2001. Proceedings of the 2001 Particle Accelerator Conference (Cat. No. 01CH37268), Vol. 3 (IEEE, New York, 2001), pp. 19031905.

[13] C. Jing, S. Gold, R. Fischer, and W. Gai, Complete multipactor suppression in an X-band dielectric-loaded accelerating structure, Appl. Phys. Lett. 108, 193501 (2016).

[14] J. Power, W. Gai, S. Gold, A. Kinkead, R. Konecny, C. Jing, W. Liu, and Z. Yusof, Observation of Multipactor in an Alumina-Based Dielectric-Loaded Accelerating Structure, Phys. Rev. Lett. 92, 164801 (2004).

[15] J. Zhang, B. J. Munroe, H. Xu, M. A. Shapiro, and R. J. Temkin, High power experimental studies of hybrid photonic band gap accelerator structures, Phys. Rev. Accel. Beams 19, 081304 (2016).

[16] C. Jing, W. Gai, J. G. Power, R. Konecny, W. Liu, S. H. Gold, A. K. Kinkead, S. G. Tantawi, V. Dolgashev, and A. Kanareykin, Progress toward externally powered $\mathrm{x}$-band dielectric-loaded accelerating structures, IEEE Trans. Plasma Sci. 38, 1354 (2010).

[17] S. Aisenberg and R. Chabot, Ion-beam deposition of thin films of diamondlike carbon, J. Appl. Phys. 42, 2953 (1971).

[18] H. Nakamori, Y. Yoshida, and K. Suzuki, The characteristic of the diamond-like carbon film prepared by the ion beam plating method using negative pulse bias, Trans. Inst. Electr. Eng. Jpn. A 122, 274 (2002).

[19] M. Noda and M. Umeno, Coating of dlc film by pulsed discharge plasma CVD, Diam. Relat. Mater. 14, 1791 (2005).

[20] Y. Yamamoto, E. Cenni, A. Four, E. Kako, S. Michizono, and Y. Okii, Recent results for study of ceramic and copper plating for power couplers, in 29th Linear Accelerator Conf.(LINAC'18), Beijing, China, 16-21 September 2018 (JACOW Publishing, Geneva, Switzerland, 2019), pp. 905-907.

[21] H. Xu, M. Shapiro, and R. J. Temkin, Measurement of internal dark current in a $17 \mathrm{GHz}$, high gradient accelerator structure, Phys. Rev. Accel. Beams 22, 021002 (2019).

[22] B. Meyerson and F. Smith, Electrical and optical properties of hydrogenated amorphous carbon films, J. Non-Cryst. Solids 35, 435 (1980).

[23] J. Halbritter, Electron loading of superconducting rf cavities, Part. Accel. 3, 163 (1972). 
[24] S. Mori, M. Yoshida, and J. D. Satoh, The design optimization of the dielectric assist accelerating structure for better heat and gas transfer, in 10th Int. Partile Accelerator Conf.(IPAC'19), Melbourne, Australia, 19-24 May 2019
(JACOW Publishing, Geneva, Switzerland, 2019), pp. 1179-1181.

[25] Y. Wei and A. Grudiev, Investigations into x-band dielectric disk accelerating structures for future linear accelerators, arXiv:2006.07276. 